\title{
TTR
}

Traduction, terminologie, re?daction

\section{La traduction comme exégèse : le cas de la poésie}

\section{Maurice Pergnier}

Volume 12, numéro 2, 2e semestre 1999

Poésie, cognition, traduction II - Autour d'un poème de W. H. Auden

Poetry, Cognition, Translation II — On a Poem by W. H. Auden

URI : https://id.erudit.org/iderudit/037377ar

DOI : https://doi.org/10.7202/037377ar

Aller au sommaire du numéro

\section{Éditeur(s)}

Association canadienne de traductologie

ISSN

0835-8443 (imprimé)

1708-2188 (numérique)

Découvrir la revue

Citer cet article

Pergnier, M. (1999). La traduction comme exégèse : le cas de la poésie. TTR, 12(2), 159-172. https://doi.org/10.7202/037377ar

\section{Résumé de l'article}

La traduction comme exégèse : le cas de la poésie - À partir de la comparaison de deux traductions d'un sonnet de Michel-Ange (La Notte), cet article étudie la relation des segments du poème à la structuration symbolique globale qui en constitue le sens le plus essentiel en même temps que le plus fermé à l'exégèse. Il essaie d'éclairer comment le rendu de chaque segment est tributaire de la bonne appréhension de cette structuration symbolique sous-jacente. À travers la réussite et les échecs de la traduction, c'est une problématique du sens en poésie qui est esquissée.
Tous droits réservés @ C TTR: traduction, terminologie, rédaction — Les auteurs, 1999
Ce document est protégé par la loi sur le droit d'auteur. L’utilisation des services d'Érudit (y compris la reproduction) est assujettie à sa politique d'utilisation que vous pouvez consulter en ligne.

https://apropos.erudit.org/fr/usagers/politique-dutilisation/ 


\section{La traduction comme exégèse : le cas de la poésie}

\section{Maurice Pergnier}

Il existe, chez les théoriciens de la traduction, une réticence certaine à intégrer les problèmes de la traduction poétique dans la réflexion générale sur les mécanismes de la traduction. Par son caractère spécifique, la poésie paraît à beaucoup échapper aux lois générales. Cette communication voudrait montrer que, non seulement la traduction de la poésie n'échappe pas à la problématique générale et relève de la même théorisation, mais qu'en outre elle est plus à même que d'autres types de traduction, par la relation particulière que le langage y entretient avec le sens, de mettre à jour certains aspects fondamentaux du processus exégétique de l'opération traduisante.

Nous nous proposons de le faire apparaître à partir d'une réflexion sur la traduction en français d'un sonnet de Michel-Ange intitulé La Notte.

Nous présentons le poème accompagné de deux de ses traductions françaises. Cette comparaison vise à montrer comment la lecture peut être tributaire d'idées préconçues projetées sur une cuvre, et en obscurcir la véritable signification.

Toute traduction - surtout d'une cuvre poétique - est une exégèse'. La valeur attribuée aux mots du poème et la justesse de ceux qui seront choisis pour les rendre dans l'autre langue dépendent de la saisie du sens et de la symbolique globale du texte. C'est par rapport à

\footnotetext{
' Pour notre définition précise du mot « exégèse » en théorie de la traduction, voir Pergnier, 1978.
} 
ce sens global que chaque détail ponctuel prend sa valeur, et que l'exactitude de la traduction de chaque segment peut être jugée.

De ce point de vue, les deux traductions considérées servent de révélateur de la résistance que le poème oppose à l'exégèse.

Le lecteur ne manquera pas d'être frappé par les nombreuses divergences entre les deux versions de cette même œuvre, divergences qui portent parfois sur des détails, mais parfois aussi sur des points essentiels - et qui apparaîtront plus essentiels encore qu'il ne semble de prime abord. Ce sont surtout à ces divergences qu'on demandera au lecteur de prêter attention, car ce sont elles qui éclairent la façon dont l'œuvre résiste à livrer d'emblée son sens. Il y a en effet tout lieu de postuler que si les traductions divergent, c'est qu'elles échouent à saisir le vrai sens de l'œuvre, ou du moins n'en livrent chacune qu'un aspect.

Cette façon de comparer des traductions pourra paraitre cruelle pour les traducteurs, puisqu'elle met en relief davantage des déficiences que des réussites. Il faut donc souligner, au préalable, que ces deux traductions ne manquent pas de mérites. Chacun, selon ses goûts, sera libre de préférer l'une ou l'autre, mais on ne peut qu'apprécier leur souci de rendre le poème "au plus près ", dans une forme française poétique, rythmée, utilisant des allitérations et assonances qui rappellent celles de l'original, et en s'efforçant à l'économie qui caractérise ici l'écriture de Michel-Ange (même si l'une apparait plus "bavarde" et plus explicative que l'autre). Certaines de leurs formulations sont obscures, mais certains passages du poème original ne le sont-ils pas aussi?

On pourra, certes, mettre ces déficits sur le compte de la difficulté inhérente à la traduction poétique et sur l'impossibilité bien réelle - de rendre toutes les nuances sémantiques d'un poème; nous pensons, quant à nous, que - dans le cas présent - les divergences constatées ne font, en fait, que refléter et révéler en les formulant les erreurs d'interprétation qui menacent tout lecteur de ce sonnet.

Ce qui est en jeu, ce n'est pas la qualité de la reformulation, c'est la qualité de l'exégèse sous-jacente (et implicite) sur laquelle elle repose. 
Certes, une partie des divergences constatées dans la formulation tient au souci des deux traducteurs de produire des versions françaises elles-mêmes poétiques, et d'assujettir le choix des mots aux nécessités du rythme, de l'assonance, voire - dans certains cas - de la rime. Ce ne sont, évidemment, pas ces différences de traitement qui nous intéresseront, mais celles qui mettent en jeu la structure signifiante globale.

Toute traduction - quelles que soient, par ailleurs, ses qualités - livre, non le sens du poème (ou de ses segments), mais l'exégèse et l'interprétation qu'un lecteur privilégié (le traducteur) en a faites $^{2}$. Alors que le poème original laisse la porte ouverte à toutes les virtualités d'interprétation, les traductions, quant à elles, "figent " de manière immuable l'exégèse qu'en a faite le traducteur.

Il va nous apparaître que les deux traducteurs (comme, sans doute, la plupart des lecteurs-exégètes) sont restés à distance de ce qui constitue le sens et, pourrait-on dire, le principe organisateur du poème. Ce faisant, ils se sont condamnés à en traduire les segments successifs comme autant d'éléments autonomes dont l'opacité reste rebelle à l'explicitation.

Cela est particulièrement vrai du deuxième vers du deuxième quatrain, dont l'importance est considérable pour l'interprétation d'ensemble du poème, et sur lequel il convient donc d'attirer d'emblée l'attention :

Che l'umid' ombra e ogni quiet' appalta,

rendu respectivement par

car l'humide pénombre dispense le repos, (G.R.-D.)

et par

l'ombre rafraichissante et paisible l'assume (P.L.)

${ }^{2}$ Cette interprétation apparaît bien dans les traductions divergentes que les deux traducteurs font du titre ( $\dot{a}$ la nuit/sur la nuit). Par ailleurs, en mettant une majuscule à Nuit G.R.-D. indique clairement qu'il perçoit celle-ci comme une allégorie. 
Le lecteur ne peut manquer d'être frappé, non seulement par la divergence d'interprétation, mais - à travers elle - par l'incongruité apparente de la notation descriptive l'umid ombra dont chaque traducteur a cherché à rendre compte à sa façon, en évitant le plat mot à mot (l'ombre humide) et en visant à échapper à l'impression de banalité qui pourrait s'en dégager.

Ce faisant, ils ont, l'un et l'autre, fermé la porte à une saisie de la valeur propre de ce segment dans la structure symbolique globale du poème: ce n'est, en effet, qu'en prenant acte de cette apparente incongruité, et en se refusant à en faire une interprétation réductrice, qu'on peut accéder à sa signifiance, dont la portée dépasse de loin le vers dans lequel elle se rencontre.

Malgré les apparences - et notamment la forme du sonnet dans lequel il se coule dans le plus pur respect des règles - ce poème de Michel-Ange est atypique. La tentation est forte - pour le lecteur, comme pour le traducteur qui l'aide à y pénétrer - de ramener l'inconnu au connu, et donc de traiter ce sonnet comme un représentant d'un genre, dont le mode de fonctionnement tant rhétorique que symbolique est familier et bien établi. Ce qui échappe aux lois du genre sera donc, inconsciemment, gommé ou ramené à l'application des perceptions conventionnelles.

C'est oublier que Michel-Ange est un génie sans pareil, et que - même si, pour lui, la poésie est un mode d'expression secondaire (un violon d'Ingres?) - il ne transporte pas moins dans ce mode d'expression l'originalité d'une personnalité exceptionnelle. Il est même permis de penser qu'un artiste accablé de tâches créatrices dans son domaine propre n'aurait pas pris la peine de travailler les mots, s'il s'était agi de broder sur de simples concetti (divertissement de beaux esprits de cour), et s'il n'avait eu à exprimer quelque chose que seule la forme poétique lui permettait d'exprimer.

Plutôt que de ramener de force le poème à une intelligibilité qu'on pourrait dire " de convention », il y a donc lieu de s'interroger sur tout ce qui, au contraire, frappe par la singularité, voir l'incongruité, et tout ce qui résiste à une interprétation trop facile, à la lumière d'une symbolique pré-établie.

On n'aura, bien sûr, pas manqué de remarquer que le poème se présente comme un éloge de la nuit, et qu'il est construit autour du 
contraste entre le jour, synonyme de souffrances, de travail et de maladie, et la nuit porteuse de toutes les bénédictions et de tous les délices. Mais on aura, peut-être, été moins attentif au fait que la nuit n'en est pas le véritable sujet. Sous couvert de la nuit, c'est en fait du sommeil et du rêve qu'il est fait l'éloge. C'est le sommeil, et non la nuit en tant que telle, qui Con pace ogn'opra sempr' al fin assalta. C'est lui qui met fin à ogni stanco pensiero. C'est lui qui efface toutes les blessures de la vie et transforme les corps malades en corps glorieux (dernier tercet). C'est lui, surtout, qui, par le rêve (in sogno) nous arrache à ce bas monde pour nous transporter " aux plus hautes régions " (alla più alta) où ni ennui ni colère n'ont plus cours. Bref, le paradis!

Le titre (surtout dans les traductions françaises qui veulent l'expliciter en l'étoffant), et l'apostrophe initiale, masquent le fait que la nuit n'est ici que le point de départ d'une méditation qui fera successivement l'éloge du sommeil, puis du rêve, et enfin de la mort (dont le sommeil est l'« ombre " = la semblance), pour culminer sur un éloge des réalités supérieures et transcendantes auxquelles cette dernière permet d'accéder.

Mal lu, le poème peut aisément être pris pour une formulation de l'idée banale : la nuit est digne d'éloges parce qu'elle nous repose de nos fatigues et nous console de nos chagrins. C'est faire fi de ce que disent explicitement certains passages, et notamment de la fin du dernier quatrain (ov'ire spero): la nuit, nous dit le poète, nous transporte en rêve de cet ici-bas méprisable (l'infima parte) aux « plus hautes régions auxquelles j'aspire... "

Le début du vers qui suit immédiatement ( $O$ ombra del morir) n'est pas moins explicite, si on veut bien le regarder pour lui-même, et non à travers des lunettes empruntées à d'autres auteurs.

La parenté du sommeil et de la mort a été maintes fois soulignée et utilisée comme motif poétique, avec une connotation généralement négative. C'est tout le contraire chez Michel-Ange : c'est parce qu'elle est ombra del morir ${ }^{3}$, que la nuit (entendez: le sommeil)

\footnotetext{
${ }^{3}$ On peut regretter que les traducteurs aient cru devoir rendre ombra del morir par le mot à mot ombre de la mort, car le mot "ombre " risque ici d'être compris comme signifiant « ombre portée », qui rend à la comparaison du sommeil avec la mort sa connotation négative et effrayante, alors que
} 
est l'objet des plus hautes aspirations. Si surprenant soit-il, le sens est clair : le sommeil, à travers le rêve (sogno) entrouvre les portes d'un univers de félicité auquel l'homme sage ne peut qu'aspirer. Celui qui a écrit ces vers est si conscient du caractère surprenant de son propos, qu'il a pris la précaution (deux derniers vers du premier quatrain) de souligner que ce n'est pas folie mais sagesse de faire l'éloge du sommeil (Ben ved'e ben intende chi t'esalta).

C'est par rapport à ces articulations symboliques que les mots composant le poème prennent toute leur valeur et leur poids sémantique.

Il n'y a évidemment rien de surprenant à ce qu'un poème consacré à la nuit comporte un nombre important de mots désignant l'obscurité. C'est, pourrait-on dire, la moindre des choses. Leur utilisation est, cependant, moins conventionnelle qu'il n'y paraît de prime abord, et c'est justement ce qui fait la difficulté de leur traduction.

C'est en raison de la banalité et de la simplicité même du registre dans lequel ils sont choisis par le poète qu'ils s'avèrent rebelles à une transposition directe en français.

La poésie, visant à dire des réalités non exprimées antérieurement, ne peut le faire qu'à travers la métaphore. Chaque mot $y$ exprime toujours autre chose que ce qu'il semble exprimer au premier degré. Il le fait par ses relations avec les autres mots du poème. Tout le défi de la traduction de la poésie tient à ce qu'il est impossible de rendre avec les mêmes mots de l'autre langue à la fois le sens de surface et le sens métaphorique.

Nero et ombra ont en français des correspondants qui couvrent à peu de chose près les mêmes éventails de polysémie. Or, ici, la simple transposition par leurs correspondants français est insatisfaisante, comme chaque lecteur peut en faire lui-même l'expérience ${ }^{4}$. C'est que

" ombra » signifie ici « image », " forme atténuée », « semblance ». Cependant, d'autres raisons militent en faveur de ce calque.

${ }^{4}$ L'usage particulier que la poésie fait du phénomène linguistique de la polysémie nous parât être l'une des clés essentielles de la structuration du langage poétique (Pergnier, 1997). 
la valeur sémantique de ces mots dans le poème ne se situe pas sur le plan concret mais sur le plan métaphorique. Plus exactement, elle se situe à la fois sur ces deux plans, et c'est le niveau métaphorique qui, bien que le plus important, est le plus délicat à appréhender.

Dans le premier vers, aucun des deux traducteurs ne rend nero par noir. (Et il est vrai que, dans un texte en prose, $O$ nott'..., benché nero apparaîtrait comme un truisme ou une platitude indigne d'être énoncée.) Les deux traducteurs ont sans doute essayé d'échapper à cette apparence de truisme en traduisant, l'un par obscur, l'autre par de l'ombre. En choisissant obscur, le premier ramène l'adjectif dans le champ du concret. De ce fait, il souligne encore plus le risque que le passage soit appréhendé comme une lapalissade. En traduisant par quoique de l'ombre, l'autre échappe à la platitude mais introduit un ésotérisme de forme qui n'est pas dans benché nero.

Or, Michel-Ange a bien choisi d'utiliser ici nero, et non ombra ou oscurita. On peut, certes, arguer des nécessités de la rime et du rythme, mais Michel-Ange n'aurait pas pris le risque d'énoncer un lieu commun si le mot ne s'imposait aussi pour des raisons plus profondes, notamment par rapport à ombra utilisé plus loin. ${ }^{5}$

L'obscurité, l'ombre, évoquent des états affaiblis de la lumière. Le noir, d'une part est une couleur; d'autre part, évoque l'absence totale de lumière, le contraire de la lumière, et - en filigrane - le néant. Il a, ici, une charge symbolique plus importante que sombre ou obscur.

L'umid' ombra du sixième vers constitue probablement le centre focal du poème. Les deux traducteurs y ont évité le calque direct. Cela manifeste qu'ils ont eu conscience de la nécessité d'une interprétation. Mais tous les deux ramènent cette interprétation sur un plan concret au lieu de chercher à interpréter le niveau métaphorique.

\footnotetext{
${ }^{5}$ Il est à craindre que ce vers reste à tout jamais impossible à traduire de façon satisfaisante pour une raison plus profonde: les mots qui le constituent ne comportent que deux voyelles, la voyelle o (o nott', dolce, tempo, nero), et la voyelle $e$, ce qui constitue un jeu de contrastes primaires dont la valeur sémantique est difficile à appréhender et qu'il est impossible de rendre dans une autre langue. On ne manquera pas d'être frappé par le fait que la voyelle $o$ est à l'initiale du vers et à la rime.
} 
La question est : pourquoi l'ombra est-elle umid? Regardons comment chaque traducteur y répond.

1. L'ombre rafraîchissante: même à supposer qu'elle puisse être entendue comme "rafraîchissant l'esprit», cette séquence évoque irrésistiblement l'idée d'une recherche de la fraîcheur de la nuit par opposition aux ardeurs du soleil. Sensation purement physique dont on ne voit guère comment elle peut ouvrir la voie alla più alta, objet des désirs les plus ardents du poète.

2. En traduisant par l'humide pénombre, l'autre traducteur choisit d'interpréter ombra mais non umid. Le résultat est une suggestion encore plus concrète de condition climatique. Liée à la " pénombre", l'humidité évoque ici plus des sensations désagréables de cave ou de cachot qu'un lieu idyllique. Or l'umid ombra est manifestement voulue par Michel-Ange comme une évocation bienheureuse, sensuelle certes, mais ouvrant les portes d'un univers transcendant. Le contraire de l'humidité froide.

Sans même approfondir davantage la structure symbolique du poème, on peut suggérer que, en raison de sa charge latente de sensualité, umid serait plus adéquatement traduit par moite que par humide.

La clé de la traduction (comme de la compréhension) réside dans l'approfondissement des raisons internes au poème qui font que l'ombre est ici donnée comme umid.

On ne peut appréhender, ici, le sens du mot ombra si on perd de vue que ce qui est évoqué/invoqué à travers la nuit, c'est le sommeil, le rêve, et plus profondément, la mort et son au-delà. Ce sont bien ces renvois métaphoriques qui structurent le sens du poème. Il apparaît notamment, de plus en plus clairement, que des traductions mettant en avant les acceptions les plus concrètes (ombre rafraichissante, pénombre, ombre de la mort) sont inadaptées à transmettre ce sens.

On comprend mieux, dès lors, la répartition et la valeur respective des mots nero et ombra dans le poème.

Dans le premier vers, nero est porteur des connotations purement négatives de la nuit ("benché nero »): absence de lumière, absence d'activité (opra), potentiellement peur du néant vaste et noir 
(Baudelaire). Elle est un « trou noir » parce qu'elle est appréhendée ici comme une plongée dans le sommeil et le rêve. Tandis qu'ombra porte - au contraire - les connotations positives : elle protège, dispense la paix et, à travers elle, on accède aux plus hautes régions et à un bonheur sans partage. Elle est la porte de l'infini et du divin (un divin qui se dessine ici comme maternel, ne serait-ce que parce que notte et ombra sont tous les deux de genre féminin, tandis que nero, par son accord grammatical avec tempo, est masculin).

Il n'est pas sans importance de remarquer que c'est le même mot, ombra, qui est utilisé à deux reprises dans des contextes différents, et que cela ne saurait être fortuit.

L'umid ombra fait ressortir l'acception "obscurité " (mais sans avoir les connotations potentiellement négatives de ce dernier mot), tandis que ombra del morir fait ressortir l'idée de "image", "semblance». On n'aura garde, cependant, d'oublier que le mot renvoie dans les deux cas métaphoriquement à la même réalité : le sommeil. Il ne faut donc pas exclure que dans ombra del morir " ombra " garde sa polysémie latente, et assume également les sens concrets de " obscurite " et " abri de la lumière solaire ». Et n'oublions pas non plus - soit dit en passant - que Michel-Ange n'écrit pas ombra della morte, mais ombra del morir (nuance particulièrement difficile à rendre en français).

Bien que les traducteurs aient tous les deux traduit ombra del morir par ombre de la mort, ils ne semblent pas avoir perçu les implications de ce segment de vers quant au sens de l'ombre et de la nuit dans le reste du poème.

Il est vrai que, pour un lecteur contemporain, les associations nuit, noirceur, ombre, sommeil, mort, sont plus chargées de connotations déplaisantes qu'inspiratrices de pensées bienheureuses ( $o v$ ' ire spero). Il lui est donc difficile d'entrer dans l'univers mental de Michel-Ange, qui présente le sommeil comme bénéfique parce qu'il est l'image (la préfiguration quotidienne) de la mort. Le poème est l'expression d'une foi chrétienne profonde qui fait concevoir la mort et son ombre projetée (non pas la morte: état, mais il morir : passage) comme une naissance à la vraie vie; mais il est aussi, n'en doutons pas, la mise en mots d'une expérience personnelle du poète en relation avec le sommeil. Si la conception chrétienne, dans laquelle Michel-Ange baignait sans doute, suffit à expliquer que la mort soit donnée comme 
étant la « vraie vie ", elle n'inclut pas le passage par l'expérience sensuelle du sommeil telle que celle-ci est évoquée ici.

Le lecteur contemporain (sauf expérience mystique et foi semblable à celle de Michel-Ange) sera enclin à considérer comme quelque peu morbide l'éloge du sommeil en tant qu'expérience anticipée de la mort, et aura tendance à lire les formulations du poète à la lumière d'associations symboliques qui lui sont plus familières.

On se tromperait cependant lourdement si on prenait cette ode à la mort (à travers sa semblance : le sommeil) pour un refus et un dénigrement de la vie. Mort et vie sont ici intimement mêlées, et sont les deux faces d'un même phénomène. Les bienfaits de l'ombra del morir ne sont-ils pas dispensés selon le poète à chi ben vive?

Si le lecteur veut bien relier ces considérations, il n'aura guère de mal à tirer avec nous des conclusions sur le sens de l'umid ombra, sens fort éloigné de l'humide pénombre d'une chambre ou de l'ombre rafraichissante.

Ce lieu dans lequel, non la nuit, mais le sommeil nous emporte, lieu à la fois ombreux et moite (plus tiède à vrai dire que " frais"), qui protège de toute douleur et libère de tout labeur, peut-il être autre chose que le sein maternel retrouvé?

C'est par ce repli dans l'ombre originelle, nous dit le poète, que l'individu a une connaissance de cet état supérieur auquel il accédera en traversant la mort. Mort/naissance ne font qu'un dans ce monde du repli que l'artiste nomme la nuit et appelle de ses vœux.

Il serait aventureux de dire que Michel-Ange a produit ces images poétiques en toute conscience et qu'il développe là une philosophie construite et assumée. Les voies de la création artistique sont distinctes de celles de la raison philosophique. La question du degré de volonté consciente du poète peut donc rester ouverte sans inconvénient pour l'exégèse des signes qui nous intéressent. 11 importe seulement de souligner qu'une traduction qui ne donne pas au lecteur la possibilité d'accéder à la compréhension intuitive de ce sens - qui gouverne toute la structure du poème - passe à côté de son objet.

Ces quelques réflexions ne suffisent pas, bien sûr, à proposer une traduction qui remplacerait plus avantageusement les deux que 
nous avons comparées entre elles et avec l'original, non plus qu'elles n'épuisent l'analyse de la structure signifiante du sonnet.

Il s'agissait seulement d'essayer de montrer que la traduction de la poésie ne mettait pas en œuvre des processus fondamentalement différents de la traduction des autres "genres 》 puisque, comme pour les autres types de textes, le pivot en est l'exégèse du sens. Cependant, si l'on veut bien admettre que la poésie est en soi un mode de connaissance, et non une simple ornementation du discours, toute traduction d'un poème - comme d'ailleurs toute glose qui en est faite - soulève immanquablement la question cruciale de la nature du sens en poésie et de sa relation à la forme. Cette question, qui sans doute n'a pas fini d'agiter le monde de la linguistique, de la poétique et de la traductologie, déborde de toute évidence l'étendue du présent propos. Nous estimerons avoir atteint un but plus modeste si nous avons réussi à faire apercevoir que la saisie de ce sens, s'il relève plus de l'intuition poétique (du traducteur ou de l'exégète) que de l'analyse rationnelle, ne relève pas pour autant de la pure subjectivité.

Université Paris-XII 
O nott', o dolce tempo, benché nero, Con pace ogn'opra sempr' al fin assalta.

Ben ved' e ben intende chi t'esalta, $E$ chi t'onor, ha l'intellett' intero.

Tu mozzi e tronchi ogni stanco pensiero, Che l'umid' ombra e ogni quiet' appalta, E d'all'infima parte alla più alta In sogno spesso porti, ov' ire spero.

O ombra del morir, per cui se ferma Ogni miseri' a l'alma, al cor nemica, Ultimo delli afflitti e buon rimedio,

Tu rendi sana nostra carn'inferma, Rasciugh' i pianti, e posi ogni fatica, $E$ furi a chi ben vive ogn' ir' e tedio.

\section{Sur la nuit}

Ô Nuit, ô temps si doux quoique de l'ombre, c'est dans ta paix que cède enfin tout acte. Qu'il sait bien voir et entendre, celui qui t'exalte! Et qui te rend grâces, il est toute clairvoyance.

Tu émousses et tranches tout penser morose, car l'humide pénombre dispense le repos, et de l'infime lieu souvent, en songe, elle nous transporte aux plus hautes régions que hante mon attente.

Ô ombre de la mort, par qui se referme toute misère qui blesse l'âme et le ccur, des affligés dernier et doux remède,

tu rends la santé à notre chair malade, tu taris nos pleurs, tu reposes de toute fatigue, à l'amant de la vie tu effaces l'ennui et la colère. 
Sonnet à la nuit

Ô nuit, ô temps suave bien qu'obscur, ta paix, pour finir, a toujours raison de tout labeur; qui t'exalte a l'œil bon et l'entendement sain, c'est un esprit sans faille qui te rend le bonheur.

À toute pensée chagrine tu coupes court :

l'ombre rafraîchissante et paisible l'assume; et souvent d'ici-bas jusqu'aux nuées tu m'emportes en songe où j'ai l'espoir de parvenir un jour.

Ô ombre de la mort dans laquelle s'apaise toute détresse d'âme dont pâtit le cœur, pour l'affligé, suprême et bienfaisant remède;

tu guéris notre chair infirme, essuies nos pleurs, nous délasses de nos fatigues, et soulages les justes de toute colère et tout ennui.

traduction de Pierre Leyris

\section{Références}

PERGNIER, Maurice (1978). Les fondements sociolinguistiques de la traduction. Lille-Paris, ART-Honoré Champion; nouvelle éd. Lille, P.U.L., 1993.

PERGNIER, Maurice (1997). Du sémantique au poétique, avec Baudelaire, Cocteau, Magritte. Paris, L'Harmattan.

RÉSUMÉ : La traduction comme exégèse : le cas de la poésie partir de la comparaison de deux traductions d'un sonnet de MichelAnge ( $L a$ Notte), cet article étudie la relation des segments du poème à la structuration symbolique globale qui en constitue le sens le plus essentiel en même temps que le plus fermé à l'exégèse. Il essaie d'éclairer comment le rendu de chaque segment est tributaire de la bonne appréhension de cette structuration symbolique sous-jacente. À travers la réussite et les échecs de la traduction, c'est une problématique du sens en poésie qui est esquissée. 
ABSTRACT : Translation as an Exegetic Procedure : The Case of Poetry : - A comparative analysis of two French translations of one of Michelangelo's sonnets (La Notte), lays particular emphasis on the links between separate segments of the poem and its symbolic context and structure. This analysis leads to an attempt to demonstrate how the translation of each of the segments is dependent upon the correct apprehension of this underlying symbolic structure. In examining the trials and errors of translation, the problematic of meaning in poetry is underlined.

Maurice Pergnier : Université de Paris Val de Marne, Faculté des Lettres et des Sciences Humaines, av. du Général de Gaulle, 94010 Créteil Cédex. 